International Journal of Modern Physics D Vol. 24, No. 14 (2015) 1599001 (12 pages)

(C) World Scientific Publishing Company

DOI: $10.1142 /$ S0218271815990011

\title{
Cumulative author index
}

Abbas, G., see Jawad

Abdalla, E., see Lin

Abebe, A., Breaking the cosmological background degeneracy by two-fluid perturbations in $f(R)$ gravity

Adamo, T., General relativity as a two-dimensional CFT

Adler, S. L. \& Ramazanoğlu, F. M., Spherically symmetric vacuum solutions arising from trace dynamics modifications to gravitation

Afshordi, N., Mann, R. B. \& Pourhasan, R., A holographic big bang?

Ahmed, N., see Rahaman

Alexandre, J., Houston, N. \& Mavromatos, N. E., Inflation via gravitino condensation in dynamically broken supergravity

Aly, A. A., Fekry, M. \& Mansour, H., Entropycorrected new agegraphic dark energy model in the context of Chern-Simons modified gravity

Ameen, A., see Borkar

Amelino-Camelia,

G., Arzano, M., Gubitosi, G. \& Magueijo, J., Gravity as the breakdown of conformal invariance

Amelino-Camelia, G. \& Astuti, V., Misleading inferences from discretization of empty spacetime: Snyder-noncommutativity case study

Amin, M. A., Hertzberg, M. P., Kaiser, D. I. \&
24 (2015) 1550061

24 (2015) 1550038

24 (2015) 1550053

24 (2015) 1544024

$24(2015) 1550011$

24 (2015) 1544029

24 (2015) 1550049

$24(2015) 1541004$

24 (2015) 1550044

24 (2015) 1550019

24 (2015) 1543002

24 (2015) 1550073
Karouby, J., Nonperturbative dynamics of reheating after inflation: A review

Amirfakhrian, M., see Pedram

Anderson, J. D., see Feldman

Andria, R., see Irakli

Araujo, A. \& Pereira, J. G., Entropy in locally-de Sitter spacetimes

Arraut, I., On the black holes in alternative theories of gravity: The case of nonlinear massive gravity

Arzano, M., see AmelinoCamelia

Astuti, V., see AmelinoCamelia

Axenides, M., Floratos, E. \& Nicolis, S., Chaotic information processing by extremal black holes

Balakin, A. B. \& Zayats, A. E., Nonminimal black holes with regular electric field

Bandyopadhyay, P., see Mullick

Banerjee, A., see Bhar

Banerjee, S., Bera, S. \& Singh, T. P., Cosmological constant, quantum measurement and the problem of time

Banik, P., see Ghosh

Banks, T., The temperature/entropy connection for horizons, massless particle scattering, and the origin of locality

Barceló, C., CarballoRubio, R. \& Garay, L. J., Uncovering the effective
24 (2015) 1530003

$24(2015) 1550016$

24 (2015) 1550066

24 (2015) 1550042

24 (2015) 1550099

24 (2015) 1550022

24 (2015) 1543002

24 (2015) 1550073

24 (2015) 1542012

24 (2015) 1542009

24 (2015) 1550074

24 (2015) 1550034

24 (2015) 1544011

24 (2015) 1550084

24 (2015) 1544010 
spacetime: Lessons from the effective field theory rationale

Barrow, J. D. \& Graham, A. A. H., New singularities in unexpected places

Bayaskar, S. N., see Katore

Beck, S., The youngest globular clusters

Belotsky, K., Khlopov, M., Kouvaris, C. \& Laletin, M., High-energy positrons and gamma radiation from decaying constituents of a two-component dark atom model

Belotsky, K. M., Esipova, E. A., Khlopov, M. Yu. \& Laletin, M. N., Dark Coulomb binding of heavy neutrinos of fourth family

Belotsky, K. M., Kirillov, A. A. \& Rubin, S. G., Primordial black holes and the observable features of the universe

Benone, C. L., Crispino, L. C. B., Herdeiro, C. A. R. \& Radu, E., Stationary bound states of massless scalar fields around black holes and black hole analogues

Bera, S., see Banerjee

Bergshoeff, E., Merbis, W., Routh, A. J. \& Townsend, P. K., The third way to 3D gravity

Bhar, P. \& Banerjee, A., Stability of thin-shell wormholes from noncommutative BTZ black hole

Bhattacharya, S. \& Shankaranarayanan, S., How emergent is gravity?

Bianchi, E., Hackl, L. \& Yokomizo, N., Entanglement time in the primordial universe

Biswas, S. Kr. \& Chakraborty, S., Interacting dark energy in $f(T)$ cosmology: A dynamical system analysis

Bjerrum-Bohr, N. E. J., Donoghue, J. F., El-

24 (2015) 1544019

24 (2015) 1544012

24 (2015) 1550017

24 (2015) 1530002

24 (2015) 1545004

24 (2015) 1545008

24 (2015) 1545005

24 (2015) 1542018

24 (2015) 1544011

24 (2015) 1544015

24 (2015) 1550034

24 (2015) 1544005

24 (2015) 1544006

24 (2015) 1550046
Menoufi, B. K., Holstein, B. R., Planté, L. \& Vanhove, P., The equivalence principle in a quantum world

24 (2015) 1544013

Blázquez-Salcedo, J. L., Extremal rotating black holes in EinsteinMaxwell-Chern-Simons theory: Radially excited solutions and nonuniqueness

Bochicchio, M., Yang-Mills mass gap at large- $N$, noncommutative YM theory, topological quantum field theory and hyperfiniteness

Bolotin, Y. L., Kostenko, A., Lemets, O. A. \& Yerokhin, D. A., Cosmological evolution with interaction between dark energy and dark matter

Boos, J., PlebańskiDemiański solution of general relativity and its expressions quadratic and cubic in curvature: Analogies to electromagnetism

Borkar, M. S. \& Ameen, A., Evaluation of Bianchi type $V I_{0}$ magnetized anisotropic dark energy models with constant deceleration parameter in bimetric theory of gravitation

Borštnik, N. M. \& Rosina, M., Are superheavy stable quark clusters viable candidates for the dark matter?

Bouhmadi-López, M., Errahmani, A., Martín-Moruno, P., Ouali, T. \& Tavakoli, Y., The little sibling of the big rip singularity

Bragança, E. A. F., Mota, H. F. S. \& de Mello, E. R. B., Induced vacuum bosonic current by magnetic flux in a higher
24 (2015) 1542016

24 (2015) 1530017

24 (2015) 1550079

24 (2015) 1550019

24 (2015) 1545003

24 (2015) 1550078
24 (2015) 1530007 
dimensional compactified cosmic string spacetime

Brandenberger, R. H., see Moghaddam

Bucher, M., Physics of the cosmic microwave background anisotropy

Bucher, M. \& Ni, W.-T., General relativity and cosmology

Buettner, D. J., see Morley

Cai, R.-G., Guo, Z.-K. \& Tang, B., Updated reduced CMB data and constraints on cosmological parameters

Cai, Y.-F., see Moghaddam

Camanho, X. O., Edelstein, J. D. \& Zhiboedov, A., Weakly coupled gravity beyond general relativity

Campuzano, C., Cordero, R., Cruz, M. \& Rojas, E., On the flat spacetime Galileons and the BornInfeld type structures

$\mathrm{Cao}, \mathrm{Z}$., see $\mathrm{He}$

Capozziello, S., De Laurentis, M. \& Luongo, O., Connecting early and late universe by $f(R)$ gravity

Carballo-Rubio, R., see Barceló

Cardoso, V., see Macedo

Carlip, S., Chiou, D.-W., Ni, W.-T. \& Woodard, R., Quantum gravity: A brief history of ideas and some prospects

Cerdonio, M. \& Rovelli, C., Casimir effects are not an experimental demonstration that free vacuum gravitates: Connections to the cosmological constant problem

Chacón-Acosta, G. \& Hernandez-Hernandez, H. H., Polymer quantum effects on compact stars models

Chakraborty, S., see Biswas

Chamel, N., On the Lie subalgebra of Killing-Milne and Killing-Cartan vector fields in Newtonian spacetime

Chan, R., see Goldoni

24 (2015) 1550082

Chen, C.-M., Nester, J. M. \& Tung, R.-S., Gravitational energy for GR and

24(2015) 1530004 Poincaré gauge theories: A covariant hamiltonian approach

24 (2015) 1530030

24 (2015) 1550004

Chen, G.-R., Zhou, S. \& Huang, Y.-C., Vector particles tunneling from BTZ black holes

Chen, J., see Huang

Chen, J.-B., Liu, Z.-Q. \&

24 (2015) 1550071

24 (2015) 1550082 Xing, L., Cosmological constraints on the variable modified Chaplygin gas model by using MCMC approach

24 (2015) 1544031 Cheng, C. \& Huang, Q.G., Constraint on inflation model from BICEP2 and WMAP 9-year data

24 (2015) 1550018

24 (2015) 1550021

24 (2015) 1530026

24 (2015) 1550005

24 (2015) 1550054

24 (2015) 1550059

24 (2015) 1550041

24 (2015) 1550081

24 (2015) 1541002

24 (2015) 1544019

24 (2015) 1542023

24 (2015) 1530028

24 (2015) 1544020

24 (2015) 1550033

24 (2015) 1550046

Chernoff, D. F. \& Tye, S.-H. H., Inflation, string theory and cosmic strings

Chilambwe, B., Hansraj, S. \& Maharaj, S. D., New models for perfect fluids in EGB gravity

Chiou, D.-W., Loop quantum gravity

Chiou, D.-W., see Carlip

Chluba, J., Hamann, J. \& Patil, S. P., Features and new physical scales in primordial observables: Theory and observation

Chowdhury, B. D., Limitations of holography

Cianci, R., see Piedipalumbo

Contopoulos, I. G., Esposito, F. P., Kleidis, K., Papadopoulos, D. B. \& Witten, L., Generating solutions to the EinsteinMaxwell equations

Cooperstock, F. I., The essence of gravitational waves and energy

Cordero, R., see Campuzano

Corichi, A., Energy of test objects on black
24 (2015) 1530010

24 (2015) 1550051

24 (2015) 1530005

24 (2015) 1530028

24 (2015) 1530023

24 (2015) 1550008

24 (2015) 1550100

24 (2015) 1550101

24 (2015) 1543005

24 (2015) 1550041
24 (2015) 1541001 
hole spacetimes: A brief review

Crispino, L. C. B., see Benone

Crispino, L. C. B., see Macedo

Cruz, M., see Campuzano

Cudell, J. R. \& Khlopov, M., Dark atoms with nuclear shell: A status review

Culetu, H., Nonsingular black hole with a nonlinear electric source

Czinner, V. G., Black hole entropy and the zeroth law of thermodynamics

Das, S., Bose-Einstein condensation as an alternative to inflation

Das, T. K., see Tarafdar

Das, U. \& Mukhopadhyay, B., Imprint of modified Einstein's gravity on white dwarfs: Unifying Type Ia Supernovae

da Silva, J. M. H., see de Brito

da Silva, M. F. A., see Goldoni

da Silva, P. M. L. T., see de Brito

de Brito, G. P., da Silva, J. M. H., da Silva, P. M. L. T. \& de Souza Dutra, A., Smooth braneworld models possibility in modified gravities

De Laurentis, M., see Capozziello

de Mello, E. R. B., see Bragança

de Souza Dutra, A., see de Brito

Degollado, J. C., Electromagnetic and gravitational signatures of accretion into black holes

Deng, X.-M., The second post-Newtonian light propagation and its astrometric measurement in the solar system

Dent, J. B., Review of the possible role of self-ordering scalar fields in production of a stochastic background of gravitational waves

di Serego Alighieri, S., Cosmic polarization rotation: An astrophysical test of fundamental physics

Ding, X., Li, Z. \& Zhu, Z.H., Toward cosmologicalmodel-independent calibrations for the luminosity relations of Gamma-Ray Bursts

Dmitriev, A. E., see Gani

Doğru, M. U. \& Yilmaz,

24 (2015) 1542015 İ., Expanding and nonexpanding conformal wormholes in scalar-tensor the-

24 (2015) 1544001

24 (2015) 1550096 ory

24 (2015) 1541005

$24(2015) 1530016$

24 (2015) 1550057

24 (2015) 1545001

Donoghue, J. F., see Bjerrum-Bohr

24 (2015) 1550064

24 (2015) 1544013

Drobov, I. V., Paklin, N. N. \& Tegai, S. Ph., A toy cosmological model with dynamical backreaction

24 (2015) 1544026

24 (2015) 1550089

24 (2015) 1550021

24 (2015) 1550089

24 (2015) 1550089

24 (2015) 1541002

24 (2015) 1550055

24 (2015) 1550089

24 (2015) 1542004

Dymnikova, I., Electromagnetic source for the KerrNewman geometry

Dymnikova, I. \& Khlopov, M., Regular black hole remnants and graviatoms with de Sitter interior as heavy dark matter candidates probing inhomogeneity of early universe

Dzhunushaliev, V., Folomeev, V. \& Urazalina, A., Star-plus-wormhole systems with two interacting scalar fields

24 (2015) 1550097

Ebadi, H. \& Moradpour, H., Thermodynamics of universe with a varying dark energy component

Edelstein, J. D., see Camanho

Ellis, H. G., Cosmology without Einstein's assumption that inertial mass produces gravity

24 (2015) 1550035

24 (2015) 1550094

24 (2015) 1545002

El-Menoufi, B. K., see Bjerrum-Bohr

Errahmani, A., see Bouhmadi-López
$24(2015) 1550098$

24 (2015) 1544031

24 (2015) 1550069

24 (2015) 1544013

24 (2015) 1550078 
Esipova, E. A., see Belotsky

Esposito, F. P., see Contopoulos

Fabris, J. C., see Tavakoli

Fekry, M., see Aly

Feldman, M. R. \& Anderson, J. D., On the possible onset of the Pioneer anomaly

Fernando, S., Regular black holes in de Sitter universe: Scalar field perturbations and quasinormal modes

Ferreira, E. G. M., see Moghaddam

Ferreira, H. R. C., Renormalized vacuum polarization of rotating black holes

Flachi, A., Black holes as QCD laboratories

Floratos, E., see Axenides

Folomeev, V., see Dzhunushaliev

Freidel, L., Leigh, R. G. \& Minic, D., Modular spacetime

Futamase, T., Gravitational lensing in cosmology

Gani, V. A., Dmitriev, A. E. \& Rubin, S. G., Deformed compact extra space as dark matter candidate

Gao, Y., see Meng

Garattini, R., Traversable wormholes in distorted gravity

Garay, L. J., see Barceló

Garny, M., Ibarra, A. \& Vogl, S., Signatures of Majorana dark matter with $t$-channel mediators

Gasperini, M., Cosmology and short-distance gravity

Ghosh, S. \& Banik, P., Characteristics of transonic spherical symmetric accretion flow in Schwarzschild-de Sitter and Schwarzschild antide Sitter backgrounds, in pseudo-general relativistic paradigm

Gillies, G. T., see Unnikrishnan
24 (2015) 1545008

24 (2015) 1550101

24 (2015) 1550062

24 (2015) 1550044

24 (2015) 1550066

24 (2015) 1550104

24 (2015) 1550082

24 (2015) 1542007

24 (2015) 1542017

24 (2015) 1542012

24 (2015) 1550097

24 (2015) 1544028

24 (2015) 1530011

24 (2015) 1545001

24 (2015) 1530029

24 (2015) 1542025

24 (2015) 1544019

24 (2015) 1530019

24 (2015) 1544003

24 (2015) 1550084

24 (2015) 1544009
Goldoni, O., da Silva, M. F. A., Chan, R. \& Pinheiro, G., Vaidya solution in general covariant Hořava-Lifshitz gravity with the minimum coupling and without projectability: Infrared limit

Gómez-Valent, A., see Solà

Govender, M., see Thirukkanesh

Graham, A. A. H., see Barrow

Greenwood, E., Timedependent particle production and particle number in cosmological de Sitter space

Grenzebach, A., Perlick, V. \& Lämmerzahl, C., Photon regions and shadows of accelerated black holes

Grobov, A. V. \& Rubin, S. G., Formation and search of large scale antimatter regions

Gruppuso, A. \& Sagnotti, A., Observational hints of a pre-inflationary scale?

Gubitosi, G., see AmelinoCamelia

Guiot, E., Alternative classical force of gravitation: A study of the free fall motion

Guiot, E., An alternative classical force of gravitation in order to explain the velocity curve of spiral galaxies

Guo, Z.-K., see Cai

Gupta, V., Kabir, R., Mukherjee, A. \& Lohiya, D., Dynamics of a scalar field, with a double exponential potential, interacting with dark matter

Habibi, F., Shaghaghian, M. \& Pazhouhesh, R., Time evolution of accreting magnetofluid around a compact objectNewtonian analysis

Hackl, L., see Bianchi

Hamann, J., see Chluba
24 (2015) 1550021

24 (2015) 1541003

24 (2015) 1550002

24 (2015) 1544012

24 (2015) 1550031

24 (2015) 1542024

24 (2015) 1550093

24 (2015) 1544008

24 (2015) 1543002

24 (2015) 1550088

24 (2015) 1550036

24 (2015) 1550071

24 (2015) 1550068

24 (2015) 1550077

24 (2015) 1544006

24 (2015) 1530023 
Han, Z., see Meng

Hansraj, S., see Chilambwe

Harko, T., Lobo, F. S. N. \& Mak, M. K., Wormhole geometries supported by quark matter at ultrahigh densities

Haskell, B. \& Melatos, A., Models of pulsar glitches

Hatkar, S. P., see Katore

He, X. \& Cao, Z., New Bondi-type outgoing boundary condition for the Einstein equations with cosmological constant

Hehl, F. W., see Heinicke

Heinicke, C. \& Hehl, F. W., Schwarzschild and Kerr solutions of Einstein's field equation: An introduction

Hendi, S. H., Lovelock black holes in the presence of nonlinear electrodynamics

Herdeiro, C. A. R., see Benone

Herdeiro, C. A. R. \& Radu, E., Asymptotically flat black holes with scalar hair: A review

Herdeiro, C. A. R. \& Radu, E., How fast can a black hole rotate?

Hernandez-Hernandez, $\mathrm{H}$. H., see Chacón-Acosta

Hertzberg, M. P., see Amin

Hod, S., Ten shades of black

Holstein, B. R., see BjerrumBohr

Honarvaryan, M., Sheykhi, A. \& Moradpour, H., Thermodynamical description of the ghost dark energy model

Hossain, Md. W., Myrzakulov, R., Sami, M. \& Saridakis, E. N., Unification of inflation and dark energy à la quintessential inflation

Houston, N., see Alexandre

Huang, Q., Chen, J. \& Wang, Y., Critical escape velocity
24 (2015) 1530029 24 (2015) 1550051

24 (2015) 1550006

24 (2015) 1530008

24 (2015) 1550017

24 (2015) 1550081

24 (2015) 1530006

24 (2015) 1530006

24 (2015) 1550040

24 (2015) 1542018

24 (2015) 1542014

24 (2015) 1544022

24 (2015) 1550033

24 (2015) 1530003

24 (2015) 1544007

24 (2015) 1544013

24 (2015) 1550048

24 (2015) 1530014

24 (2015) 1541004 for a charged particle in

Ernst spacetime

24 (2015) 1550054

24 (2015) 1541001

24 (2015) 1550005

24 (2015) 1530019

24 (2015) 1550003

Ikram, A, see Sharif

Iorio, A., Curved spacetimes and curved graphene: A status report of the Weyl symmetry approach

Iorio, L., Gravitational anomalies in the solar system?

Iorio, L., Post-Newtonian direct and mixed orbital effects due to the oblateness of the central body

Irakli, G., Zaza, O. \& Andria, $\mathrm{R}$., On the role of rotation in the outflows of the Crab pulsar

Ita, E. E., Four-dimensional gravity as an almostPoisson system

Jalalzadeh, S. \& Rostami, T., Covariant extrinsic gravity and the geometric origin of dark energy

Jawad, A. \& Abbas, G., Interacting new agegraphic version of pilgrim dark energy

Jones, P. \& Singleton, D., Gravitons to photons Attenuation of gravitational waves

Jou, D., Sciacca, M. \& Mongiovì, M. S., Thermal duality and thermodynamics of micro black holes

Juárez-Aubry, B. A., Can a particle detector cross a Cauchy horizon?

Kabir, R., see Gupta

Kaiser, D. I., see Amin

Kane, G., Sinha, K. \& Watson, S., Cosmological moduli and the postinflationary universe: A critical review

Kapoor, R., Kar, S. \& Singh, D., Quantum effects in topological and Schwarzschild de Sitter
24 (2015) 1530013

24 (2015) 1530015

24 (2015) 1550067

24 (2015) 1550042

24 (2015) 1550047

$24(2015) 1550027$

$24(2015) 1550061$

$24(2015) 1544017$

24 (2015) 1550087

24 (2015) 1542005

24 (2015) 1550068

24 (2015) 1530003

24 (2015) 1530022 
brane: Aspects of torsion on $(D \bar{D})_{4}$-brane

Kar, S., see Kapoor

Karbasi, S. \& Razmi, H., Spherical "Top-Hat" collapse in a modified Chaplygin gas dominated universe

Karouby, J., see Amin

Katore, S. D., Hatkar, S. P. \& Bayaskar, S. N., Some special solutions in Bianchi type $\mathrm{VI}_{0}$ cosmological models with modified chaplygin gas in general relativity

Khlopov, M., see Belotsky

Khlopov, M., see Cudell

Khlopov, M., see Dymnikova

Khlopov, M. Yu., see Belotsky

Khoshabadi, S., see Rastegarzadeh

Kirillov, A. A., see Belotsky

Kleidis, K., see Contopoulos

Kleihaus, B., Kunz, J. \& Radu, E., Black Ringoid in seven dimensions

Kostenko, A., see Bolotin

Kouvaris, C., see Belotsky

Koyama, K., see Sakstein

Kraus, P. \& Mathur, S. D., Nature abhors a horizon

Kuhfittig, P. K. F., Macroscopic traversable wormholes with zero tidal forces inspired by noncommutative geometry

Kunz, J., see Kleihaus

Kuroda, K., Ground-based gravitational-wave detectors

Kuroda, K., Ni, W.-T. \& Pan, W.-P., Gravitational waves: Classification, methods of detection, sensitivities and sources

Laletin, M., see Belotsky

Laletin, M. N., see Belotsky

Lämmerzahl, C., see Grenzebach

Landim, R. C. G., Coupled tachyonic dark energy: A dynamical analysis
Lanzafame, G., Collective molecular dissipation on Navier-Stokes macroscopic scales: Accretion disc viscous modeling in $\mathrm{SPH}$

Lebed, A. G., Breakdown of the equivalence between gravitational mass and energy for a quantum body: Theory and suggested experiments

Leigh, R. G., see Freidel

Lemets, O. A., see Bolotin

Lemos, J. P. S., see Luz

Lemos, J. P. S., Lopes, F. J.

24 (2015) 1550017

24 (2015) 1545004

24 (2015) 1545007

24 (2015) 1545002

24 (2015) 1545008

24 (2015) 1550080

24 (2015) 1545005

24 (2015) 1550101

24 (2015) 1542019

24 (2015) 1530007

24 (2015) 1545004

24 (2015) 1544021

24 (2015) 1543003

24 (2015) 1550023

24 (2015) 1542019

24 (2015) 1530032

24 (2015) 1530031

24 (2015) 1545004

24 (2015) 1545008

24 (2015) 1542024

24 (2015) 1550085 \& Minamitsuji, M., Rotating thin shells in $(2+1)$ dimensional asymptotically AdS spacetimes: Mechanical properties, machian effects, and energy conditions

Letellier, C., see Messager

Li, Z., see Ding

Lima, J. A. S., Perico, E. L. D. \& Zilioti, G. J. M., Decaying vacuum inflationary cosmologies: Searching for a complete scenario including curvature effects

Lin, K., Abdalla, E. \& Wang, A., Holographic superconductors in Hořava-Lifshitz gravity

Liu, Z.-Q., see Chen

Lobo, F. S. N., see Harko

Lohiya, D., see Gupta

Lopes, F. J., see Lemos

Lortan, D. B., see Thirukkanesh

Luongo, O., see Capozziello

Luz, P. \& Lemos, J. P. S., Newtonian wormholes with spherical symmetry and tidal forces on test particles

Macedo, C. F. B., Crispino, L. C. B., Cardoso, V., Okawa, H. \& Pani, P., Evidence for event horizons: Long-lived modes in ultracompact objects

Magueijo, J., see AmelinoCamelia
24 (2015) 1550028

24 (2015) 1530027

24 (2015) 1544028

24 (2015) 1530007

24 (2015) 1542020

24 (2015) 1542022

24 (2015) 1530024

24 (2015) 1550057

24 (2015) 1541006

24 (2015) 1550038

24 (2015) 1550059

24 (2015) 1550006

24 (2015) 1550068

24 (2015) 1542022

24 (2015) 1550002

24 (2015) 1541002

24 (2015) 1542020

24 (2015) 1542023

24 (2015) 1543002 
Maharaj, S. D., see Chilambwe

Mak, M. K., see Harko

Makukov, M. A., see Mychelkin

Malinovsky, A., see Shatskiy

Manchester, R. N., Pulsars and gravity

Mandanici, G., On the invariance of the relative rest in doubly special relativity

Mann, R. B., see Afshordi

Mansour, H., see Aly

Martín-Moruno, P., see Bouhmadi-López

Martín-Moruno, P. \& Nunes, N. J., Accelerating universe as a result of an adjustment mechanism

Martinec, E. J., Fractionated branes and black hole interiors

Marto, J., Tavakoli, Y. \& Moniz, P. V., Improved dynamics and gravitational collapse of tachyon field coupled with a barotropic fluid

Mas, J. \& Serantes, A., Oscillating shells in Anti-de Sitter space

Masi, N., On the behavior of the bounds of the holographic theory for massive and massless particle systems

Masoudi, M. \& Saffari, R., Study of a quadratic redshift-based correction in $f(R)$ gravity with Baryonic matter

Masoumi, A., State of matter at high density and entropy bounds

Mathur, S. D., see Kraus

Mavromatos, N. E., see Alexandre

Melatos, A., see Haskell

Mena, F. C., Cylindrically symmetric models of gravitational collapse to black holes: A short review

Meng, X., Gao, Y. \& Han, Z., $\mathrm{SNe}$ Ia as a cosmological probe
Merbis, W., see Bergshoeff

24 (2015) 1550051

24 (2015) 1550006

24 (2015) 1544025

24 (2015) 1550075

24 (2015) 1530018

24 (2015) 1550012

24 (2015) 1544029

24 (2015) 1550044

24 (2015) 1550078

24 (2015) 1544018

24 (2015) 1543004

24 (2015) 1550025

24 (2015) 1542003

24 (2015) 1550076

$24(2015) 1550091$

24 (2015) 1544016

24 (2015) 1543003

24 (2015) 1541004

24 (2015) 1530008

24 (2015) 1542021

24 (2015) 1530029
Messager, V. \& Letellier, C., A genesis of special relativity

Mielke, E. W., Mass gap in Yang's theory of gravity

Mignemi, S., Classical dynamics on Snyder spacetime

Minamitsuji, M., see Lemos

Minic, D., see Freidel

Mirza, B., see Poshteh

Mitra, A., When can an "Expanding Universe" look "Static" and vice versa: A comprehensive study

Moghaddam, H. B., Brandenberger, R. H., Cai, Y.F. \& Ferreira, E. G. M., Parametric resonance of entropy perturbations in massless preheating

Moglie, E. D., see Piedipalumbo

Mongiovì, M. S., see Jou

Moniz, P. V., see Marto

Monteiro, R., O'Connell, D. \& White, C. D., Gravity as a double copy of gauge theory: From amplitudes to black holes

Moon, T., see Myung

Moradpour, H., see Ebadi

Moradpour, H., see Honarvaryan

Morley, P. D. \& Buettner, D. J., SHM of galaxies embedded within condensed neutrino matter

Mota, H. F. S., see Bragança

Moura, F., On the temperature dependence of the absorption cross-section for black holes in string theory

Mukherjee, A., see Gupta

Mukhopadhyay, B., see Das

Mullick, L. \& Bandyopadhyay, P., Fermions, qdeformed diffeomorphism and the evolution of spin networks

Mychelkin, E. G. \& Makukov, M. A., Fundamental scalar fields and the dark side of the universe
24 (2015) 1544015

$24(2015) 1530024$

24 (2015) 1550070

24 (2015) 1550043

24 (2015) 1542022

24 (2015) 1544028

24 (2015) 1550029

$24(2015) 1550032$

24 (2015) 1550082

24 (2015) 1550100

24 (2015) 1550087

24 (2015) 1550025

24 (2015) 1542008

24 (2015) 1550095

24 (2015) 1550098

$24(2015) 1550048$

24 (2015) 1550004

24 (2015) 1550055

24 (2015) 1542011

24 (2015) 1550068

24 (2015) 1544026

24 (2015) 1550074

24 (2015) 1544025 
Myrzakulov, R., see Hossain

Myung, Y. S. \& Moon, T., Scale-invariant scalar spectrum from the nonminimal derivative coupling with fourth-order term

Nashed, G. G. L., KerrNewman solution in modified teleparallel theory of gravity

Nemati, M., see Rastegarzadeh

Nesseris, S. \& Sapone, D., Novel null-test for the $\Lambda$ cold dark matter model with growth-rate data

Nester, J. M., see Chen

Ni, W.-T., see Bucher

Ni, W.-T., see Carlip

Ni, W.-T., see Kuroda

Nicolis, S., see Axenides

Novikov, I. D., see Shatskiy

Nunes, N. J., see MartínMoruno

Oboudiat, F., see Poshteh

O'Connell, D., see Monteiro

Okawa, H., see Macedo

Olmo, G. J. \& RubieraGarcia, D., The quantum, the geon and the crystal

Ouali, T., see BouhmadiLópez

Padmanabhan, T., Gravity in six elegant steps

Paklin, N. N., see Drobov

Pal, S., Rajeev, K. \& Shankaranarayanan, S., An approach to the quantization of black hole quasinormal modes

Pan, W.-P., see Kuroda

Pani, P., see Macedo

Panigrahi, D., Thermodynamical behavior of the variable Chaplygin gas

Papadopoulos, D. B., see Contopoulos

Parikh, M., Two roads to the null energy condition

Patil, S. P., see Chluba

Pazhouhesh, R., see Habibi

Pedram, P., Amirfakhrian, M. \& Shababi, H., On the $(2+1)$-dimensional
24 (2015) 1530014

24 (2015) 1550095

24 (2015) 1550007

24 (2015) 1550010

24 (2015) 1550045

24 (2015) 1530026

24 (2015) 1530030

24 (2015) 1530028

24 (2015) 1530031

24 (2015) 1542012

24 (2015) 1550075

24 (2015) 1544018

24 (2015) 1550029

24 (2015) 1542008

24 (2015) 1542023

24 (2015) 1542013

24 (2015) 1550078

24 (2015) 1544002

24 (2015) 1550035

24 (2015) 1550083

24 (2015) 1530031

24 (2015) 1542023

24 (2015) 1550030

24 (2015) 1550101

24 (2015) 1544030

24 (2015) 1530023

24 (2015) 1550077
Dirac equation in a constant magnetic field with a minimal length uncertainty

Pereira, J. G., see Araujo

Perico, E. L. D., see Lima

Perlick, V., see Grenzebach

Perlick, V., see Philipp

Philipp, D. \& Perlick, V., Schwarzschild radial perturbations in EddingtonFinkelstein and PainlevéGullstrand coordinates

Piedipalumbo, E., Moglie, E. D. \& Cianci, R., Updated $f(T)$ gravity constraints from high-redshift cosmography

Pinheiro, G., see Goldoni

Planté, L., see Bjerrum-Bohr

Poshteh, M. B. J., Mirza, B. \& Oboudiat, F., Generalized Ehrenfest's equations and phase transition in black holes

Pourhasan, R., see Afshordi

Pradhan, A., see Rahaman

Pradhan, P., Horizon straddling ISCOs in spherically symmetric string black holes

Quevedo, H., Quevedo, M. N. \& Sánchez, A., Thermodynamics and geometrothermodynamics of Born-Infeld black holes with cosmological constant

Quevedo, M. N., see Quevedo

Radu, E., see Benone

Radu, E., see Herdeiro

Radu, E., see Herdeiro

Radu, E., see Kleihaus

Rahaman, F., Pradhan, A., Ahmed, N., Ray, S., Saha, B. \& Rahaman, M., Fluid sphere: Stability problem and dimensional constraint

Rahaman, M., see Rahaman

Rahvar, S., Gravitational microlensing I: A unique astrophysical tool

Rajeev, K., see Pal
24 (2015) 1550016

24 (2015) 1550099

24 (2015) 1541006

24 (2015) 1542024

24 (2015) 1542006

24 (2015) 1542006

24 (2015) 1550100

24 (2015) 1550021

24 (2015) 1544013

24 (2015) 1550029

24 (2015) 1544029

24 (2015) 1550049

24 (2015) 1550086

24 (2015) 1550092

24 (2015) 1550092

24 (2015) 1542018

24 (2015) 1542014

24 (2015) 1544022

24 (2015) 1542019

24 (2015) 1550049

24 (2015) 1550049

24 (2015) 1530020

24 (2015) 1550083 
Ramazanoğlu, F. M., see Adler

Ranea-Sandoval, I. F. \& Vucetich, H., Scalar resonances in axially symmetric spacetimes

Rastegarzadeh, G. \& Khoshabadi, S., Mass discrimination using the inferred depth of maximum through the particle densities measured at observation level

Rastegarzadeh, G. \& Nemati, M., Dependence of the muon pseudorapidity on the cosmic ray mass composition around the knee

Ray, S., see Rahaman

Razmi, H., see Karbasi

Rocha, J. V., Gravitational collapse with rotating thin shells and cosmic censorship

Rojas, E., see Campuzano

Ropotenko, K., Energy scale of inflation from the recurrence time of the universe

Rosina, M., see Borštnik

Rosswog, S., The multimessenger picture of compact binary mergers

Rostami, T., see Jalalzadeh

Routh, A. J., see Bergshoeff

Rovelli, C., see Cerdonio

Rowland, D. R., On claims that general relativity differs from Newtonian physics for self-gravitating dusts in the low velocity, weak field limit

Rubiera-Garcia, D., see Olmo

Rubin, S. G., see Belotsky

Rubin, S. G., see Gani

Rubin, S. G., see Grobov

Rudra, P., Towards a possible solution for the coincidence problem: $f(G)$ gravity as background

Ruggiero, M. L., Gravitoelectromagnetic effects of massive rings

Saffari, R., see Masoudi
24 (2015) 1550011

24 (2015) 1550037

24 (2015) 1550080

24 (2015) 1550010

24 (2015) 1550049

24 (2015) 1550050

24 (2015) 1542002

24 (2015) 1550041

24 (2015) 1550026

24 (2015) 1545003

24 (2015) 1530012

24 (2015) 1550027

24 (2015) 1544015

24 (2015) 1544020

24 (2015) 1550065

24 (2015) 1542013

24 (2015) 1545005

24 (2015) 1545001

24 (2015) 1550093

24 (2015) 1550013

24 (2015) 1550060 24 (2015) 1550091
Sagnotti, A., see Gruppuso

Saha, B., see Rahaman

Sakhelashvili, O., Dimensional reduction in 6D standing waves braneworld

Sakstein, J. \& Koyama, K., Testing the Vainshtein mechanism using stars and galaxies

Samain, E., Clock comparison based on laser ranging technologies

Sami, M., see Hossain

Sánchez, A., see Quevedo

Sapone, D., see Nesseris

Saridakis, E. N., see Hossain

Sato, K. \& Yokoyama, J., Inflationary cosmology: First $30+$ years

Schee, J., see Stuchlík

Sciacca, M., see Jou

Sen, A., Riding gravity away from doomsday

Serantes, A., see Mas

Shababi, H., see Pedram

Shaghaghian, M., see Habibi

Shan, C.-L., Reconstructing the WIMP velocity distribution from direct dark matter detection data with a nonnegligible threshold energy

Shankaranarayanan, S., see Bhattacharya

Shankaranarayanan, S., see Pal

Sharif, M. \& Ikram, A., Wormholes supported by $f(\mathcal{G})$ gravity

Sharif, M. \& Zaeem Ul Haq Bhatti, M., Structure scalars and super-Poynting vector of tilted Szekeres geometry

Shatskiy, A., Novikov, I. D. \& Malinovsky, A., Spectrum for the electric dipole which nonradially falling into a black hole

Sheykhi, A., see Honarvaryan

Siahaan, H. M., Instability of charged massive scalar fields in bound states
24 (2015) 1544008

24 (2015) 1550049

24 (2015) 1550009

24 (2015) 1544021

24 (2015) 1530021

24 (2015) 1530014

24 (2015) 1550092

24 (2015) 1550045

24 (2015) 1530014

24 (2015) 1530025

24 (2015) 1550020

24 (2015) 1550087

24 (2015) 1544004

24 (2015) 1542003

24(2015) 1550016

24 (2015) 1550077

24 (2015) 1550090

24 (2015) 1544005

24 (2015) 1550083

24 (2015) 1550003

24 (2015) 1550014

24 (2015) 1550075

24 (2015) 1550048 
around Kerr-Sen black holes

Singh, D., see Kapoor

Singh, D. V., Power law corrections to BTZ black hole entropy

Singh, T. P., see Banerjee

Singleton, D., see Jones

Sinha, K., see Kane

Sivaram, C., Gravitational waves: Some less discussed intriguing issues

Škovran, M., Analytical solutions for cosmological perturbations in a onecomponent universe with shear stress

Solà, J., The cosmological constant and entropy problems: Mysteries of the present with profound roots in the past

Solà, J. \& Gómez-Valent, A., The $\bar{\Lambda} \mathrm{CDM}$ cosmology: From inflation to dark energy through running $\Lambda$

Stuchlík, Z. \& Schee, J., Circular geodesic of Bardeen and Ayon-Beato-Garcia regular black-hole and nohorizon spacetimes

't Hooft, G., Local conformal symmetry: The missing symmetry component for space and time

Tang, B., see Cai

Tanhayi, M. R., Particle creation in global de Sitter space: Bulk space consideration

Tarafdar, P. \& Das, T. K., Dependence of acoustic surface gravity on geometric configuration of matter for axially symmetric background flows in the Schwarzschild metric

Tavakoli, Y., see BouhmadiLópez

Tavakoli, Y., see Marto

Tavakoli, Y. \& Fabris, J. C., Creation of particles in a cyclic universe driven by loop quantum cosmology

Tegai, S. Ph., see Drobov
24 (2015) 1550102

24 (2015) 1550015

24 (2015) 1550001

24 (2015) 1544011

24 (2015) 1544017

24 (2015) 1530022

24 (2015) 1544023

24 (2015) 1550063

24 (2015) 1544027

24 (2015) 1541003

24 (2015) 1550020

24 (2015) 1543001

24 (2015) 1550071

24 (2015) 1550052

24 (2015) 1550096

24 (2015) 1550078

24 (2015) 1550025

24 (2015) 1550062 24 (2015) 1550035
Thirukkanesh, S., Govender, M. \& Lortan, D. B., Spherically symmetric static matter configurations with vanishing radial pressure

Toth, V. T., see Turyshev

Townsend, P. K., see Bergshoeff

Tung, R.-S., see Chen

Turyshev, S. G. \& Toth, V. T., New perturbative method for solving the gravitational $N$-body problem in the general theory of relativity

Tye, S.-H. H., see Chernoff

Unnikrishnan, C. S. \& Gillies, G. T., Is the Higgs mechanism true to the equivalence principle?

Urazalina, A., see Dzhunushaliev

van Putten, M. H. P. M., A holographic bound on the total number of computations in the visible universe

Vanhove, P., see BjerrumBohr

Vogl, S., see Garny

Vucetich, H., see RaneaSandoval

Wall, A. C., A Second Law for higher curvature gravity

Wallemacq, Q., Composite dark matter and directsearch experiments

Wang, A., see Lin

Wang, Y., see Huang

Ward, B. F. L., Prediction for the cosmological constant in resummed quantum gravity and constraints on susy GUTs

Watson, S., see Kane

Weng, Z.-H., Dynamic of astrophysical jets in the complex octonion space

Wesson, P. S., The status of modern five-dimensional gravity (A short review: Why physics needs the fifth dimension)

White, C. D., see Monteiro
24 (2015) 1550002 24 (2015) 1550039

24 (2015) 1544015

24 (2015) 1530026

24 (2015) 1550039

24 (2015) 1530010

24 (2015) 1544009

24 (2015) 1550097

24 (2015) 1550024

24 (2015) 1544013

24 (2015) 1530019

$24(2015) 1550037$

24 (2015) 1544014

24 (2015) 1545006

24 (2015) 1550038

24 (2015) 1550054

24 (2015) 1530009

24 (2015) 1530022

24 (2015) 1550072

24 (2015) 1530001 24 (2015) 1542008 
Willison, S., Quasilinear reformulation of Lovelock gravity

Witten, L., see Contopoulos

Woodard, R., see Carlip

Xing, L., see Chen

Yang, Q., Axions/Axion-like particles and the CMB asymmetric dipole

Yerokhin, D. A., see Bolotin

Yilmaz, I., see Dog̃ru

Yokomizo, N., see Bianchi
Yokoyama, J., see Sato

Zaeem Ul Haq Bhatti, M.,

24 (2015) 1542010 see Sharif

Zayats, A. E., see Balakin

Zaza, O., see Irakli

Zhao, X.-F., The properties of the massive neutron star PSR J0348+0432

24 (2015) 1545009

24 (2015) 1530007

24 (2015) 1550064

24 (2015) 1544006
24 (2015) 1530025

24 (2015) 1550014

24 (2015) 1542009

24 (2015) 1550042

24 (2015) 1550058

24 (2015) 1544031

24 (2015) 1550005

24 (2015) 1550057

24 (2015) 1541006 